\title{
Linx
}

Revue des linguistes de l'université Paris X Nanterre

5 | 1994

La négation

\section{Destin lacanien de ladiscordance et de la forclusion}

Michel Arrivé

\section{OpenEdition}

Journals

Édition électronique

URL : http://journals.openedition.org/linx/1187

DOI : 10.4000/linx.1187

ISSN : 2118-9692

Éditeur

Presses universitaires de Paris Nanterre

\section{Édition imprimée}

Date de publication : 1 juin 1994

Pagination : 11-26

ISSN : 0246-8743

\section{Référence électronique}

Michel Arrivé, "Destin lacanien de ladiscordance et de la forclusion », Linx [En ligne], 5 | 1994, mis en ligne le 18 juillet 2012, consulté le 01 mai 2019. URL : http://journals.openedition.org/linx/1187 ; DOI 10.4000/linx.1187

Ce document a été généré automatiquement le 1 mai 2019.

Département de Sciences du langage, Université Paris Ouest 


\title{
Destin lacanien de ladiscordance et de la forclusion
}

\author{
Michel Arrivé
}

1 Je me vois contraint de prendre, à la hâte, trois précautions :

1) Contrairement à la plupart des intervenants en ce colloque, je ne suis pas un spécialiste de la négation.

2) Je ne vais d'ailleurs pas parler de la négation: comment le pourrais-je, puisque «la notion de négation est en réalité absente de la pensée-langage du français »? C'est en tout cas ce que posent, sans ambages, Damourette et Pichon ([1930], p. 146).

3) A prendre les mots au sens strict, on peut même douter que je parle de linguistique. Les deux ensembles textuels que je vais alléguer ne relèvent pas de la linguistique stricto sensu : Damourette et Pichon sont les auteurs d'un Essai de grammaire de la langue française. Et Lacan, même s'il s'appuie avec précision sur plusieurs linguistes, même s'il lui arrive, çà et là de tenir - et fort bien - le discours du linguiste, ne s'intéresse pas directement à la langue en elle-même, mais à « l'inconscient structuré comme un langage » - formule où il convient de mettre en évidence, outre le comme, le un et le langage, qui, chacun à leur façon, font problème.

2 Comment, dans ces conditions, justifier ma présence en ces lieux ? Par deux raisons :

1) S'ils mettent en cause l'existence de la négation en français, Damourette et Pichon reconnaissent avec bonne grâce que la combinaison des deux «taxièmes" de la « discordance » et de la «forclusion» en fournit l'équivalent approché : parler de ces deux taxièmes, c'est bien de quelque façon parler de la négation.

2) Il faudrait une bonne dose de pessimisme, voire de nihilisme épistémologique pour poser que le discours grammatical de Damourette et Pichon, et le discours de Lacan sur l'inconscient structuré comme un langage puissent être coupés de toute relation avec la linguistique : parler de ces deux textes, c'est bien de quelque façon parler de linguistique. 
Jinsisterai peu sur la théorie damourettetpichonienne (désormais DP, tant pour le monstre dicéphale des auteurs que pour l'adjectif dérivé de leurs noms) de la négation. Elle est, sans doute, connue de tous. Dans le bref résumé que je vais en faire, je soulignerai certains éléments souvent passés sous silence, par DP eux-mêmes ou par leurs lecteurs. La réflexion de DP part de l'analyse de l'aspect formel des phrases négatives du français :

« La négation y est constituée, ainsi que chacun de nous le sait depuis l'école élémentaire, par deux morceaux, d'une part l'affonctif ne, d'autre part les affonctifs pas, rien, jamais » ([1930], p. 130) ${ }^{1}$.

6 Je ne fais que signaler que le contre-argument traditionnellement opposé à une analyse de ce type - la fréquence des énoncés, notamment oraux, où le ne n'apparaît pas - est neutralisé par DP d'une façon très autorisée. Les travaux récents sur le problème (notamment Gadet 1989 et Winther 1990) confirment, plus de soixante ans après, leurs analyses.

7 Plus ou moins directement, DP laissent entendre que cette duplicité de la négation est une spécificité et même une supériorité du français, qui marque avec «finesse» et « délicatesse » ce que les autres langues signifient avec "grossièreté, brutalité », voire « vulgarité » ([1930], p. 144). Faut-il rappeler que la négation à deux termes est loin d'être spéciale au français ? Tesnière (1959, p. 229), qui ne tarit pas d'éloges pour DP et retient l'essentiel de leur analyse, signale cependant, discrètement, que d'autres langues (l'italien de Florence, le breton) ont une négation du même type. Et Hagège (1982, p. 86) évalue à $17 \%$ le nombre des langues objets de ses fiches qui comportent une négation à deux termes. Il laisse cependant entendre avec prudence que c'est seulement dans un nombre limité de ces langues que les deux éléments ne constituent pas un morphème discontinu et sont donc pourvus, chacun, d'un signifié différent. Il ne semble pas totalement convaincu que ce soit le cas pour le français.

8 DP, pour leur part, en sont convaincus. C'est l'effet de leur postulat fondamental : tout élément matériel de la langue comporte nécessairement une contre-partie sémantique. Pour préciser l'analyse des deux éléments de la négation, ils étudient les cas où ils fonctionnent isolément. Les résultats sont les suivants :

\section{La discordance et le discordantiel}

9 Ne marque la « discordance », par exemple après les comparatifs d'inégalité :

10 «Le cas le plus clair peut-être, c'est la présence de ne après les comparatifs d'inégalité. Il est évident qu'il y a en pareil cas une discordance entre la qualité envisagée et l'étalon ( échantil) auquel on le rapporte. Aussi est-il de règle d'utiliser ne dans ce cas » ([1930], p. 131).

11 L'un des points forts de l'argumentaire de DP sur le discordantiel tient dans la quasiimpossibilité de faire apparaître ne après les comparatifs d'égalité, où il n'y a pas discordance, mais concordance entre les deux qualités comparées. Ils citent avec délectation un exemple où le locuteur efface un ne préalablement posé au moment où il corrige en comparative d'égalité une préalable comparaison d'inégalité :

12 «Je suis frappé plus que je ne le croyais ou plutôt autant que je le croyais » (M. AAG ${ }^{2}$, le 9 février 1938, [1943], p. 131). 
13 Et ils s'acquittent en virtuoses de la tâche difficile de rendre compte des cas - à vrai dire rarissimes - où le ne apparaît après une comparaison d'égalité. Ainsi, Proust fait dire à un " ascensoriste » qu' " un ouvrier est aussi bien un monsieur que ne l'est un homme du monde ». Et DP d'argumenter, dans leur style inimitable, à la fois guindé et ironique :

14 «Aura-t-on suffisamment expliqué ces exemples quand on sera venu à dire qu'il s'agit d'une copie de la tournure convenant aux accommensuratifs d'inégalité ? Non, à notre avis ; car cela n'expliquera ni pourquoi le tour est rare, ni pourquoi c'est dans tels cas particuliers qu'il est apparu. Selon nous il faut chercher une explication psychologique : il y a une manière de protestation discordantielle du locuteur. L'ascensoriste dont il est question dans l'exemple de Proust donne à son interlocuteur une leçon d'égalitarisme ; le ne marque la protestation contre l'usage des gens du monde qui n'appellent pas un chauffeur un monsieur » ([1943], p. 131-132).

15 En somme, en dépit de l'absence de discordance entre les éléments comparés, il est possible de déceler une autre discordance, mais déplacée : elle se situe entre le sujet qui parle (le sujet de l'énonciation) et celui dont l'autre discours (celui des " gens du monde ») est, dans l'analyse DP, signalé par le ne. Il y a ici véritable polyphonie: on entend simultanément le discours auquel correspondrait la phrase attendue (sans ne) et celui que fait surgir l'occurrence insolite du discordantiel. C'est, on le verra plus bas, une analyse de ce type que fait Lacan, même s'il déplace une fois de plus la discordance, non toutefois sans retenir la présence de la polyphonie de deux sujets.

16 Un autre morceau de bravoure de DP porte sur les complétives dépendant d'un verbe marquant la crainte. A propos d'un exemple d'Anatole France - «Son petit cousin se présentait au cercle. Il craignait qu'il ne fût blackboulé »- ils ont cette belle formule :

17 « Dans la crainte, il y a discordance entre le désir du sujet de la principale et la possibilité qu'il envisage » ([1930], p. 132).

18 Sans même juger utile de justifier ce qui est à leurs yeux une évidence, DP interprètent immédiatement la crainte du sujet comme le désir inverse qui lui est sous-jacent: craindre le black-boulage de son petit cousin, c'est désirer son admission. Faisons ici un second pas - facile - vers Lacan : le « désir du sujet » présuppose un " sujet du désir ». On verra la place que prend ce concept dans l'analyse lacanienne de la discordance.

19 Je me contenterai de renvoyer au texte de DP (ou aux longues citations qui en sont faites dans Arrivé et Chevalier 1970) pour les autres faits de discordance énumérés dans les volumes de [1930] et [1943]. J'en dirai seulement que les auteurs réussissent de façon généralement convaincante à repérer une discordance dans tous les cas étudiés, même s'il faut reconnaître, avec Martin, 1984, p. $100^{3}$, que les objets entre lesquels s'institue cette discordance sont quelque peu disparates. DP répondraient sans doute que peu leur en chaut : c'est la discordance qui compte, et non ce entre quoi elle s'établit.

20 Ainsi, ne n'est pas négatif par lui-même. Les cas, bien connus des grammairiens, où il a l'air de l'être (les je n'ose, je ne sais, je ne puis, il ne cesse, etc) sont expliqués, de façon à peine alambiquée, par «le sémième propre des verbes qui tolèrent ne comme négation » ([1943], p. 164). 


\section{La forclusion et les forclusifs}

21 Là encore, DP vont chercher la clef du fonctionnement de la forclusion dans les emplois où les forclusifs, hors de l' "alliance " avec le discordantiel, sont dépourvus de valeur négative. Paul Bourget, évoquant le cas d'un chirurgien vieillissant, lui prête un pronostic pessimiste sur son avenir professionnel :

«Il est peu probable que j'opère jamais plus ».

22 Dans ce type d'emploi, «on aperçoit que les idées touchées par jamais, plus, etc, sont comme expulsées du champ des possibilités aperçues par le locuteur [...] Une opération ultérieure du chirurgien est forclose du monde probable tel que ce chirurgien l'aperçoit » ([1930], p. 139).

On constate que la forclusion à laquelle ces idées sont soumises n'équivaut nullement à une négation, comme suffit à le prouver la possibilité de la substitution à jamais et plus de un jour et encore. Ainsi corrigée, la valeur référentielle de la phrase de Bourget reste intacte : c'est bien la même situation qu'elle prend en charge à titre de référent. Seule a changé l'attitude à l'égard de ce donné référentiel inchangé du sujet de l'énonciation. C'est ce que laissent entendre DP en [1943], p. 173 et sqq. Par exemple, à propos de la contrariété d'une Madame Minot :

"Je suis très contrariée que vous ayez jamais entendu parler d'elle ",

24 ils remarquent que la « locutrice voudrait bien rejeter hors du réel (souligné par MA) le fait que Jane Peck a entendu parler de Lucile Snow » (p. 175).

Et, dès [1930], ils commentent de façon très pertinente l'épisode ultime - la mort d'Esterhasy, rapportée par un journaliste - de l'affaire de l'affaire Dreyfus :

«"L'affaire Dreyfus, dit-il, c'est pour moi un livre qui est désormais clos". Il dut se repentir jusqu'à sa dernière heure de l'avoir jamais ouvert ».

D'une façon assez exceptionnelle dans l'Essai, DP vont jusqu'à faire allusion, à propos de cet exemple, aux travaux psychanalytiques de l'un d'eux :

«Le langage est pour celui qui sait en déchiffrer les images un merveilleux miroir des profondeurs de l'inconscient. Le repentir est le désir qu'une chose passée, donc irréparable, n'ait jamais existé ; la langue française, par le forclusif, exprime ce désir de scotomisation, traduisant ainsi le phénomène normal dont la scotomisation décrite en pathologie mentale par M. Laforgue et l'un de nous, est l'exagération pathologique ${ }^{4}$ ([1930], p. 140).

On voit comment se préfigure ici l'idée de l'inconscient structuré comme un langage, sous l'aspect à vrai dire inversé du langage (sous les espèces de la langue française) structuré comme l'inconscient: la forclusion, phénomène linguistique, est l' "image » de la scotomisation.

Il est sans doute bon, avant d'en venir au sort réservé par Lacan aux deux notions DP, de jeter un coup d'œil sur la fortune de ces deux notions chez les linguistes spécialistes du français, et par ce biais d'oser une manière d'appréciation de leur théorie de la négation. 
En dépit de nombreuses critiques pas toujours fondées ${ }^{5}$, la réflexion de DP sur la négation a été fréquemment utilisée (voir, plus bas, l'analyse de Tesnière), et plus souvent encore citée jusqu'aux alentours des années 60 . Elle laisse encore des traces non négligeables, notamment terminologiques, par exemple dans l'enseignement universitaire ${ }^{6}$, et jusque dans des analyses où le contenu auquel renvoie cette terminologie n'est pas nettement pris en compte (voir, ici-même, la contribution de P. Hirschbuhler et Labelle, qui utilisent constamment le mot forclusif pour désigner le second élément de la négation).

Il semble cependant qu'aujourd'hui les travaux sur la négation se passent facilement de la référence à DP. Le $n^{\circ} 62$ de Langue française sur La négation (Attal et Muller 1984) cite DP dans sa bibliographie, mais ne l'utilise guère. Le $n^{\circ} 94$ de la même revue, consacré par Callebaut 1992 aux Négations ne fait même plus apparaître DP dans sa bibliographie. Et Callebaut 1991 cite bien des articles de DP (d'ailleurs antérieurs à l'Essai), mais ne les exploite pas dans ses analyses. Détail significatif, de la part d'un bon connaisseur de l'œuvre des deux grammairiens'.

Il faut toutefois apporter à ce constat un correctif : si DP sont aujourd'hui négligés par les spécialistes de la négation, ils restent l'une des références privilégiées pour l'étude du ne " explétif ", c'est-à-dire du discordantiel dans ses emplois non négatifs. Ainsi Milner (1973, puis - à vrai dire de façon implicite - 1978, enfin - et cette fois de façon explicite 1989), Danon-Boileau (1987 et 1991), Martin (1984) retiennent, de façon évidemment diverse, la notion de discordance dans leurs analyses du ne explétif.

Ce déséquilibre est à mon sens révélateur : DP accomplissent leur programme un peu trop bien. Ils rendent compte avec pertinence des emplois isolés du discordantiel et du forclusif. Mais il faut bien avouer que leur analyse des cas où les deux éléments s' « allient » pour fournir au français sa négation est décevante, voire autocontradictoire. Je me contente ici de signaler deux points, d'ailleurs complémentaires :

34 1) On s'étonne de constater que DP n'accordent que peu d'attention à l'ordre d'occurrence du discordantiel et du forclusif. Est-ce en raison des difficultés que font apparaitre les cas où cet ordre n'est pas respecté (personne ne m'aime) ? Tesnière, toujours discrètement, corrigera cette insuffisance, sans d'ailleurs réussir à se tirer de façon pleinement satisfaisante des cas d'inversion de l'ordre habituel (voir Tesnière, 1959, p. 224-227 et Arrivé, 1994).

2) La conséquence de cette inattention à l'ordre d'intervention des deux éléments est fâcheuse. En effet, DP en viennent, en contradiction flagrante avec leurs propos initiaux, à considérer le discordantiel et le forclusif comme l'un et l'autres négatifs. C'est du moins ce qui ressort, à mes yeux, de commentaires du type suivant :

«Les phénomènes exprimés par les verbes ne seront niés - autant du moins que la langue française est capable de les nier - que par la convergence de la notion de discordance et de celle de forclusion. Ex. :

"Je n'en finirai jamais" (Alfred Jarry, Ubu roi, III, 8)

En finir n'appartient pas à la réalité temporelle que j'aperçois, et d'autre part cela serait en discordance avec cette réalité. Je ne perçois aucun signe qui permette de pronostiquer que j'en finisse (forclusif). Bien plus, que j'en finisse serait en discordance avec toute l'ambiance dans laquelle je baigne (discordantiel) » ([1930], p. 143).

La description de DP inverse l'ordre des deux éléments, comme s'il était indifférent. Le résultat est que chacun d'eux en vient, dans l'analyse qui en est faite, à prendre isolément le statut d'une quasi-négation. On a l'impression bizarre que le discordantiel ne fait 
qu'enchérir (c'est ce que semble dire le «bien plus ») sur une négation déjà réalisée par le forclusif, qui ne s'est pourtant pas encore manifesté. On observe d'autre part l'élargissement considérable de la notion de discordance, qui en vient à s'établir entre le référent visé et l' « ambiance dans laquelle baigne » le locuteur.

Serait-il possible de reprendre à nouveaux frais une théorie de la négation à l'aide des deux « taxièmes » DP ? Il faudrait sans doute pour cela les débarrasser, précisément, de leur statut de taxièmes - c'est-à-dire de simples contenus linguistiques -, et les prendre pour des marqueurs d'opérations énonciatives. Ce serait d'ailleurs conforme à certaines indications de DP, même dans les commentaires du type de celui que j'ai critiqué, où l'on aura remarqué qu'apparait, sous la forme du je, le sujet de l'énonciation. On serait alors nécessairement amené à tenir compte de l'ordre d'intervention de ces deux opérations.

La tâche, en elle-même difficile, n'est évidemment pas celle d'un amateur égaré dans un colloque sur la négation. Elle est en outre dépourvue de pertinence à l'égard du sort que Lacan réserve, séparément, à la discordance et à la forclusion.

On vient de l'apercevoir: c'est isolément l'une de l'autre que Lacan retient les deux notions DP. Il convient donc d'étudier successivement le sort qu'il a réservé à chacune d'elles. Je serai bref sur le détail philologique des références à l'une et à l'autre. Elles sont nombreuses: Pichon - souvent seul à être nommé par Lacan, qui, comme les autres analystes, aime à citer surtout ses pareils - est, peu après Saussure, le linguiste dont le nom apparait le plus souvent dans les Écrits comme dans les volumes publiés du Séminaire. En outre, il est fréquent de trouver des allusions, diversement précises, à la discordance et à la forclusion sans que les noms de leurs géniteurs soient allégués. C'est ce qu'on observe par exemples dans les Écrits (p. 372) pour la discordance et dans le Séminaire III pour la forclusion (voir plus bas). En sorte qu'il est possible à certains lecteurs de Lacan (par exemple J.D. Nasio, 1987) de consacrer (en toute bonne foi ? Je m'interroge) de longs développements à la forclusion lacanienne sans souffler mot de l'origine DP du concept. On me dira peut-être qu'après tous les mots discordance et forclusion ne sont pas la propriété de DP : ils existent dans la langue et on peut les employer sans se référer à DP. Acceptable, dans certaines limites, à propos de la discordance, la remarque est fort discutable pour la forclusion. Car le sens technique qu'a pris ce mot en matière de droit administratif est somme toute assez éloigné de celui que lui confèrent, selon son étymologie, les deux grammairiens.

\section{La discordance}

Le problème qui se pose est à l'évidence celui des objets entre lesquels, chez Lacan, s'institue la discordance. Est-ce, comme dans le discours explicite de DP (il est vrai corrigé par certains de leurs commentaires de détail), entre deux «faits » ou deux « idées »? Que non pas. Un fragment du Séminaire VII sur L'Éthique de la psychanalyse est sur ce point pleinement explicite : c'est entre l'énonciation et l'énoncé que se situe la discordance :

"J'ai déjà relevé devant vous, sur la trace de Pichon, l'usage si subtilement différencié dans la langue française de ce ne discordantiel dont je vous ai montré ce qui le fait apparaître de façon paradoxale quand, par exemple, le sujet énonce sa propre crainte. 
Je crains, non pas comme la logique semble l'indiquer, qu'il vienne - c'est bien là ce que le sujet veut dire - mais je crains qu'il ne vienne. Ce ne a sa place flottante entre les deux niveaux du graphe ${ }^{8}$ dont je vous ai appris à faire usage pour en retrouver la distinction, celui de l'énonciation et celui de l'énoncé. En énonçant je crains... quelque chose, je le fais surgir dans son existence, et du même coup dans son existence de vœu - qu'il vienne. C'est là que s'introduit ce petit ne, qui montre la discordance de l'énonciation à l'énoncé » (1986, p. 79).

41 par le je, il ne devra être repéré nulle part ailleurs que dans le ne, qui est donc la marque de son surgissement au niveau de l'énoncé. Sujet de l'énonciation à vrai dire bien 
spécifique : il n'est tel qu' « en tant que perce son désir ». «Sujet du désir », en somme, qui nous fait retrouver le "désir du sujet» allégué par DP dans leur analyse du discordantiel.

Reste naturellement à comprendre comment peut s'opérer l'identification entre les deux notions de sujet de l'énonciation et de sujet du désir: elle n'a rien d'évident pour le linguiste. Chez Lacan, elle s'effectue par l'entremise de l' "articulation signifiante ", au sens saussurien du mot articulation et au sens lacanien du mot signifiant ${ }^{12}$. A propos d'un commentaire sur le rêve il ne savait pas qu'il était mort, il remarque que " l'imparfait garde le champ radical de l'énonciation, c'est-à-dire du rapport du sujet le plus foncier avec l'articulation signifiante. C'est dire qu'il n'en est pas l'agent, mais le support, pour autant qu'il ne saurait même en supputer les conséquences. C'est dans son rapport à l'articulation signifiante que lui, comme sujet, surgit comme sa conséquence » (1986, p. 258-259).

47 Ainsi le sujet de l'énonciation n'est autre que la «conséquence» de l'articulation signifiante: point "agent", mais simple "support». On aperçoit dès lors que l'énonciation en vient à se confondre avec l'enchaînement des signifiants. C'est du moins ce que permet d'induire la confrontation du texte de L'Éthique cité plus haut avec le graphe qui y est explicitement allégué et les commentaires qui le décryptent dans le Séminaire V:

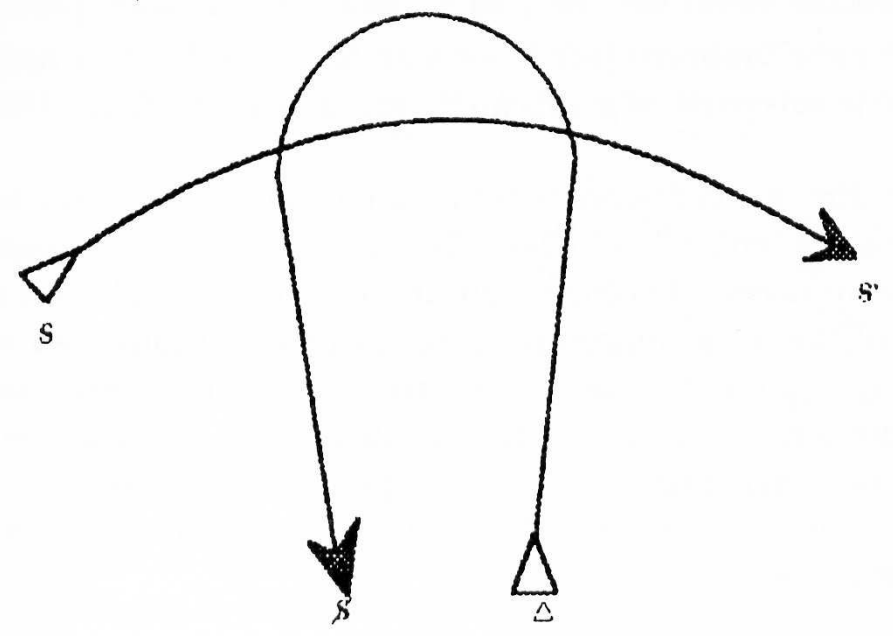

« Dans le premier temps de cette première ligne [la ligne SS', ou S est l'initiale de Signifiant, MA] nous avons la chaîne signifiante en tant qu'elle reste entièrement perméable aux effets proprement signifiants de la métaphore et de la métonymie » (6/11/1957, p. 14).

Quant à l'autre tracé (DS barré, ou S « connote ${ }^{13}$ - comme dirait Lacan - non plus le signifiant, mais le sujet barré), il est facile de voir que c'est aussi celui du signifié. C'est en effet ce qui est marqué sans équivoque par « la simple raison que [les deux tracés] glissent l'un sur l'autre » (Séminaire V, 6/11/1957, p. 15). Or on se souvient que ce "glissement » est précisément le mode de relation que Lacan, à tout moment, affecte à la relation entre 
signifiant et signifié : «la notion d'un glissement incessant du signifié sous le signifiant s'impose donc » (1966, p. 502).

On l'a compris : l'énonciation lacanienne n'est autre que celle qui enchaîne, "sur une autre scène ", ces signifiants spécifiques qui constituent l'inconscient. On aperçoit alors comment elle peut être en totale discordance avec l'énoncé, au sens que lui donnent d'une même voix Lacan et les linguistes. Quant au ne discordantiel, on comprend comment il peut rester, selon qu'il apparaît ou reste occulté, « en suspension » entre les deux niveaux du graphe :

51 «La particule négative ${ }^{14}$ ne $[n e]^{15}$ vient au jour qu'à partir du moment où je parle vraiment [sur le vecteur DS barré, MA], et non pas au moment où je suis parlé, si je suis au niveau de l'inconscient » (1986, p. 79).

Est-ce à dire que la discordance implique une indépendance totale de deux instances entre lesquelles elle s'institue? Le surgissement possible du ne au niveau de l'énoncé suffit à montrer qu'il n'en est rien. Le graphe confirme cette rencontre entre les deux niveaux : les deux vecteurs qui les représentent se croisent en deux points. C'est dans ce croisement que s'enracine la théorie lacanienne de la phrase et du discours : on sait qu'il prend le nom de " point de capiton ». Mais on s'éloignerait par trop de la discordance en entrant plus avant dans le détail de ce problème.

\section{La forclusion}

53 Tant qu'on les envisage - comme ici - du strict point de vue du linguiste, les faits sont plus simples : au contraire de la discordance - qui, par définition, intervient entre deux objets - la forclusion opère sur un objet unique. Le tout sera d'identifier cet objet.

Dans des conditions qu'il est aisé de prendre pour intentionnellement spectaculaires, Lacan fait intervenir le concept de forclusion à l'extrême fin du Séminaire III sur Les psychoses. Il le propose, au terme de la mûre réflexion qu'a constituée l'ensemble du Séminaire, pour traduire, définitivement, et même, semble-t-il, éliminer le concept freudien de Verwerfung. On sait qu'il s'agit là, dans l'appareil de Freud, de l'opération par laquelle quelque chose (mais quoi ?) est rejeté, exclu, en somme forclos. Cette Verwerfung est, dans la lecture de Lacan, fondamentalement différente de la Verdrängung (refoulement), même si les objets sur lesquels elle s'exerce sont de même nature. Lacan insiste à plusieurs reprises sur cette distinction, peut-être un peu plus labile, chez Freud, qu'il ne le laisse entendre, en traduisant $(1981 ;$ pp 22, 57, 170) de façon, a-t-on dit, un peu forcée un passage du texte freudien :

"A propos de la Verwerfung, Freud dit que le sujet ne voulait rien savoir de la castration, même au sens du refoulement. En effet, au sens du refoulement, on sait encore quelque chose de ce dont même on ne veut, d'une certaine façon, rien savoir, et c'est toute l'analyse de nous avoir montré qu'on le sait fort bien. S'il y a des choses dont le patient ne veut rien savoir, même au sens du refoulement, cela suppose un autre mécanisme. Et comme le mot Verwerfung apparaît en connexion directe avec cette phrase et aussi quelques pages auparavant, je m'en empare. Je ne tiens pas spécialement au terme, je tiens à ce qu'il veut dire, et je crois que Freud a voulu dire cela » (1981, p. 170).

On sent la légère inquiétude de Lacan: la Verwerfung freudienne n'a peut-être pas le dessin absolument net qu'il est tenté de lui attribuer au début du séminaire. Et la traduction qu'il donne du fragment de «L'homme aux loups » - texte qui fait une place 
explicite à la Verwerfung - insiste sans doute un peu trop sur le même, évidemment déterminant pour la distinction des deux opérations ${ }^{16}$. C'est sans doute la raison pour laquelle il en vient à lui substituer la forclusion : bel exemple de coalescence entre deux notions, l'une issue de l'appareil freudien, l'autre empruntée à un appareil linguistique ${ }^{17}$ :

«Il est impossible de méconnaître, dans la phénoménologie de la psychose, l'originalité du signifiant comme tel. Ce qu'il y a de tangible dans le phénomène de tout ce qui se déroule dans la psychose, c'est qu'il s'agit de l'abord par le sujet d'un signifiant comme tel, et de l'impossibilité de cet abord. Je ne reviens pas sur la notion de Verwerfung, dont je suis parti, et pour laquelle, tout bien réfléchi, je vous propose d'adopter définitivement cette traduction que je crois la meilleure - la forclusion » (1981, p. 361).

En somme, l'objet qui est soumis à la Verwerfung-forclusion, c'est « ce qui a été mis hors de la symbolisation générale structurant le sujet » (1981, p. 58). Cet objet, certes, semble différent de celui qui, chez DP, subit la forclusion. Au fait, l'est-il totalement? Et les « idées touchées par jamais ou plus, expulsées du champ des possibilités aperçues par le locuteur » (voir plus haut) ne seraient-elles pas susceptibles elles aussi d'être prises pour des signifiants? Je laisserai la question pendante, et me contenterai de l'essentiel : quoi qu'il en soit de la différence éventuelle des objets, l'opération est rigoureusement identique et mérite donc bien son nom DP de forclusion.

Reste à préciser la spécificité du signifiant ainsi soumis à la forclusion. Là encore, et d'une façon qu'on ne peut que lire comme intentionnellement spectaculaire, Lacan feint de procéder, tout au long du séminaire, à une longue enquête, qui en vient à prendre une forme quasi-policière. "Nous ne pouvons pas dire tout de suite ce qu'est ce signifiant ", pose-t-il p. 227. Plusieurs semaines plus tard, il n'est guère plus avancé, pour l'évidente raison qu' «il n'y a nul moyen de saisir au moment où cela manque, quelque chose qui manque» (p. 318). Enfin vient la réponse, à la suite d'une longue métaphore filée qui assimile le signifiant à « la grand-route $»^{18}$ :

«Quel est le signifiant qui est mis en suspens dans sa crise inaugurale? C'est le signifiant procréation dans sa forme la plus problématique, celle que Freud lui-même évoque à propos des obsessionnels, qui n'est pas la forme être mère, mais la forme être père » (p. 329).

58 A ce signifiant il ne reste plus dès lors qu'à donner son véritable nom: ce ne peut évidemment être que « le Nom-du-Père » (p. 344). C'est là le signifiant dont l'absence fait trou dans la chaîne, lacune qui en vient à « menacer tout l'édifice » (1981, p. 99).

M'attarderais-je, ludiquement, à revenir, précisément en ce point sur le silence ici observé par Lacan non seulement sur Damourette - dont l'absence, presque constante, n'a rien de pertinent - mais aussi sur Pichon lui-même, pourtant révérencieusement cité en plusieurs autres occurrences? On pourrait me dire que le mot forclusion n'est, pas plus qu'aucun autre, la propriété de ceux qui lui ont donné un sens spécifique. Après tout, le lexique juridique - auquel appartient par ailleurs le nom de la forclusion - n'est nullement étranger à Lacan, et l'on peut à la rigueur arguer que c'est cette valeur juridique du mot qui est retenue par Lacan. L'exercice toutefois est difficile. Car l'ensemble du Séminaire insiste, on l'a compris, sur l'ensemble des opérations d' " exclusion» (p. 21): Verdrängung, Verwerfung, Verleugnung, certes, mais aussi, nommément alléguée à plusieurs reprises, Verneinung. On voit même Lacan s'interroger explicitement sur les relations entre Verwerfung et Verneinung: 
« Freud admet un phénomène d'exclusion pour lequel le terme de Verwerfung paraît valable, et qui se distingue de la Verneinung, laquelle se produit à une étape très ultérieure » (1981, p. 21). forclusion soit coupé de toute relation avec celle des théories de la négation qui informent de la façon la plus insistante la réflexion de Lacan? Non, la forclusion lacanienne - au même titre que la discordance - s'enracine bien dans l'appareil DP. Et l'absence du nom de Pichon n'y est pas innocente. Irai-je à parler de forclusion du « Nomdu-Père » ? Ce serait s'interroger sur le statut de Pichon par rapport à Lacan... Faut-il dire que je ne le ferai pas? le champ de l'inconscient une opération préalablement installée dans celui du langage. On ne s'étonnera donc pas de lui voir rencontrer, quoique de façon inversée, la réflexion de DP, qui voyaient dans le mécanisme linguistique de la forclusion la «traduction » d'une opération de l'inconscient: on relira sur ce point le texte cité plus haut où ils allèguent l'article de Laforgue et Pichon.

62 J'ai pleinement conscience, au moment de conclure cet exposé, de m'être assez considérablement éloigné du thème du colloque. C'est à peine si j'aurai parlé de la négation, disjointe qu'elle est, chez DP, entre les deux taxièmes de la discordance et de la forclusion. Bien plus: j'aurai été contraint de suivre Lacan dans le chemin qu'il m'imposait: celui de l'inconscient. Mais me serai-je pour autant éloigné du langage? Point du tout, si l'on se souvient que «l'inconscient est structuré comme un langage ». Et l'on voit, par l'exemple qui a été choisi - il en est, certes, beaucoup d'autres - qu'il est possible, en suivant fidèlement la filière DP-Lacan, de donner un sens précis à cet axiome fondamental de la réflexion lacanienne.

\section{NOTES}

1. Bizarrement, DP omettent ici le «etc» dont ils accompagnent habituellement ce type d'énumération.

2. Il ne s'agit ni d'un nom, ni d'une abréviation, mais de l'immatriculation que DP ont fait subir à chacun des innombrables sujets dont ils ont utilisé les propos à titre d'exemples.

3. Les critiques de Martin sont, de son point de vue "logico-sémantique " (que sa première composante disqualifierait aux yeux de DP), pertinentes à l'égard de tous les cas examinés, à la seule réserve des comparatives d'inégalité, qui lui arrachent un aveu d'impuissance, et même l'entraînent à une bizarre contradiction : après avoir récusé, p. 106, « la thèse de l'accord », il en vient, p. 113, à « penser que ne s'explique sans plus par un fait d'accord».

4. Un appel de note renvoie à la référence bibliographique attendue : «E. Pichon et R. Laforgue, "La notion de Schizonoïa", Le Rêve et la psychanalyse, pp. 207 et 208 ». L'ouvrage est paru en 1926 chez Maloine, et l'article se trouve aux pages 173 à 210 . 
5. Je pense notamment à celle de Meillet, qu'on devine agacé par l'irruption dans le champ de la linguistique de ces deux amateurs. Il va jusqu'à leur prêter l'idée que «ne garde une valeur négative" (1931,p. 152), ce qui est évidemment en contradiction absolue avec les propos des auteurs. L'essentiel du texte de Meillet est cité dans Arrivé et Chevalier 1970, p. 155-156.

6. Entre de nombreux autres exemples possibles, voir Soutet, 1989, p. 85.

7. Callebaut est en effet l'auteur d'une bonne bibliographie de DP (Callebaut 1983), toutefois surpassée par celle, ultérieure, de Hélène Huot 1991.

8. Il s'agit du graphe qui, dans les Écrits servira à « présenter où se situe le désir par rapport à un sujet défini de son articulation par le signifiant" (1966, p. 805). Il sera, ici, introduit plus bas.

9. Lacan traduit, plus vigoureusement, Spaltung par « division, déchirure » (1986, p. 122).

10. Ces conditions ont été décrites de façon très éclairante par Culioli, 1990, p. 152-153.

11. On trouve là l'origine du jeu de mots qui transformera le discours courant en disque ourcourant.

12. Faut-il préciser que ce n'est pas ici le lieu de poser le problème - compliqué - des relations entre le signifiant saussurien et son homonyme lacanien? Je me contente de l'approximation consistant à les disjoindre, non toutefois sans insister sur ce que signale entre eux de commun le fait qu'ils sont l'un et l'autre « articulés ».

13. On aura remarqué que Lacan a pour tic lexical à peu près constant d'utiliser "connoter » pour « signifier », verbe que, visiblement, il n'aime pas.

14. On remarque qu'ici Lacan s'écarte de la lettre de l'enseignement DP et traite le ne comme négatif.

15. L'édition du Séminaire rend Lacan coupable d'une belle haplologie (ici corrigée entre crochets) qui s'établit entre le ne du discours et son propre autonyme. Comme s'il n'y avait pas de métalangage...

16. Je n'ai malheureusement pas le texte allemand sous les yeux, et il n'est même pas certain que le passage que j'ai repéré (1979, p. 389) soit effectivement celui que traduit Lacan, d'ailleurs de façon évolutive : car, p. 21, il substitue à au sens du refoulement un au sens du refoulé, qui n'en est pas l'exact équivalent. Si le fragment est correctement identifié, il faut bien avouer que les deux traducteurs - Marie Bonaparte et Rudolph. M. Lœwenstein - ne lisent pas le texte de la même façon que Lacan... Ce n'est pas à dire qu'ils aient raison!

17. On se souvient que la relation établie par Lacan entre la Vorstellungsrepräsentanz freudienne et le signifiant saussurien donne un autre exemple d'une semblable coalescence.

18. Sic dans l'orthographe adoptée par l'éditeur.

\section{RÉSUMÉS}

La théorie de la négation mise en place par Damourette et Pichon dans l'Essai de grammaire de la langue française pose que la négation n'existe pas dans la «pensée-langage » du français. La langue s'est constitué un équivalent approché de la négation en combinant deux éléments : le discordantiel ne et la série des forclusifs pas, point, jamais, rien, personne, etc. La discordance marquée par le discordantiel s'établit entre "deux idées ou deux faits » tels qu'ils sont envisagés par le sujet. La forclusion marquée par les forclusifs est une opération consistant à rejeter (forclore) hors du réel l'objet notionnel affecté.

Lacan, lecteur attentif de Damourette et Pichon, leur emprunte les deux notions, non sans les déplacer considérablement. La discordance s'établit pour lui entre l'énoncé et l'énonciation, cette 
dernière notion étant préalablement redéfinie par lui dans le cadre de la théorie de "l'inconscient structuré comme un langage". Quant à la forclusion, elle intervient dans la description de la psychose, et constitue l'équivalent lacanien de la Verwerfung freudienne.

Les faits étudiés donnent un exemple entre tous instructif de la façon dont les procédures à l'œuvre dans le langage peuvent être utilisées pour décrire les mécanismes de l'inconscient.

\section{AUTEUR}

\section{MICHEL ARRIVÉ}

Paris X 